\title{
ANALYSIS OF AN INCOMPLETE TWO-WAY
}

RESPONSE DATA

\section{Chihiro HIROTSU}

(Faculty of Engineering, University of Tokyo)

\section{SUMMARY}

Suppose that we have response data to questionaires on two subjects $A$ and $B$, say and that some of them are incomplete in the sense that they are blank on $A, B$ or both of $A$ and $B$. Then there arise two problems to be considered.

(1) Is the degree of association between $A$ and $B$ among complete data likely to be the same with that among incomplete data?

(2) If (1) is true how can we use the incomplete data to analyze the overall assosiation between $A$ and $B$ ?

Main results on these problems are stated in the following section.

\section{MAIN RESULTS IN A TWO-WAY CASE}

Let $y_{i j k l}, i=1, \cdots, a ; j=1, \cdots, b ; k=1,2 ; l=1,2$, be the frequency in the $i$ th category of $A$ and $j$ th category of $B$, where $k$ is 1 or 2 according as there is a response to $A$ or not and the suffix $Z$ is similarly defined with respect to $B$. Then data available are $y_{i j 11}, y_{i \cdot 12}, y_{j 21}$ and $y_{.22}$, where we use the usual dot notation to express the sum of variables with respect to the suffix replaced by dot.

We assume the $y_{i j k}$ to be distibuted as independent Poisson variables with the mean $m_{i j k l}$ and consider the log-1inear mode1, 


$$
\begin{aligned}
& \log m_{i j k l}=k+\alpha_{i}+\beta_{j}+\gamma_{l}+\delta_{m}+(\alpha \beta)_{i j}+(\alpha \gamma)_{i Z} \\
& +(\alpha \delta)_{i m}+(\beta \gamma)_{j l}+(\beta \delta)_{j m}+(\gamma \delta)_{\eta m}+(\alpha \beta \gamma)_{i j l} \\
& +(\alpha \beta \delta)_{i j m}+(\alpha \gamma \delta)_{i Z m}+(\beta \gamma \delta)_{j Z m}+(\alpha \beta \gamma \delta)_{i j \eta m}
\end{aligned}
$$

The parameters in the right-hand side of (1) are defined to be zero if any one of four suffixes equals one.

Theorem 1 Parameters in the model

$$
\begin{aligned}
\log m_{i j k l}=k & +\alpha_{i}+\beta_{j}+\gamma_{\eta}+\delta_{m}+(\alpha \beta)_{i j} \\
& +(\alpha \delta)_{i m}+(\beta \gamma)_{j l}+(\gamma \delta)_{Z m}
\end{aligned}
$$

are estimable whereas those in the model (3) are not all estimable unless $a=b$,

$$
\begin{aligned}
\log m_{i j k \ell}=\kappa & +\alpha_{i}+\beta_{j}+\gamma_{\eta}+\delta_{m}+(\alpha \beta)_{i j} \\
& +(\alpha \gamma)_{i Z}+(\beta \delta)_{j m}+(\gamma \delta)_{\eta_{m}}
\end{aligned}
$$

It should be noted that the number of parameters in models (2) and (3) coincides with the number of data available.

Theorem 2 Setting $(\gamma \delta)_{Z_{m}}=0$ in (2), we can test for the model

$$
\begin{aligned}
\log m_{i j k l}=\kappa & +\alpha_{i}+\beta_{j}+\gamma_{l}+\delta_{m}+(\alpha \beta)_{i j} \\
& +(\alpha \delta)_{i m}+(\beta \gamma)_{j l}
\end{aligned}
$$

by the method of maximum 1ik1ihood.

Models (2) and (4) mean that the degree of association between $A$ and $B$ is free from the pattern of the occurences of no response.

If (4) is true we can test for the hypotheses $(\alpha \beta)_{i j}=0,(\alpha \delta)_{i m}=0$ or $(\beta \gamma)_{j l}=0$ by the method of maximum liklihood.

We have similar results in higher-way cases. 